\title{
Editorial to the Special Issue: Uncertainty Quantification and Multiple-Scale Methods for Porous Media
}

\author{
Mohaddeseh Mousavi Nezhad $^{1} \cdot$ Mohammad Rezania $^{1} \cdot$ Vahid Joekar-Niasar $^{2}$
}

Published online: 19 December 2018

(c) Springer Nature B.V. 2018

Modelling flow and solute transport through porous media often involves identifying and dealing with considerable uncertainties that are mostly rooted in the heterogeneities of the media and also the different probable reaction processes. Realistic modelling and prediction of transport in porous media is essential for many different engineering works ranging from landfills and waste disposal facilities, to agricultural irrigation or pesticide applications, subsurface carbon dioxide sequestration and even biomedical engineering (Nezhad 2010). Given the widespread practical implications of the prediction of flow in porous media, during the past few decades, a significant number of different experimental and numerical studies have been carried out on the identification, characterization, quantification and evaluation of the randomness (subsurface hydrological) or (of the transport) processes and, as a result, many different stochastic methodologies have been proposed (e.g., Dagan and Neuman 1997; Riva et al. 2001; Nezhad and Javadi 2011). The developments made have highlighted the underlying complexities in modelling of multi-phase flow and reactive transport mechanisms through different porous media and the requirement to develop practical frameworks effective in capturing the true description of the porous media behavior and genuine capacity for application. The approaches needed span a wide range of topics and mechanisms including appropriate selection of random variables, development of hydraulic and mechanical constitutive models, formulation of the relevant coupled effects among possible thermo-hydro-chemo-mechanical processes, suitable spatial and temporal discretization, and numerical implementation algorithms.

This special issue is devoted to uncertainty quantification and multiple-scale methods for porous media, the subject of a minisymposium that was organized in the 9th International Conference on Porous Media held in Rotterdam, Netherlands, May 8-11, 2017. The papers included in this special issue provide an in-depth insight into several of the themes addressed in this minisymposium session. It is evident that there is not a unified approach that can be used to capture the complex behavior of porous media under different conditions; that is, different approaches should be used for different scenarios and different scale problems. However,

Mohaddeseh Mousavi Nezhad m.mousavi-nezhad@warwick.ac.uk

1 School of Engineering, The University of Warwick, Coventry CV4 7AL, UK

2 School of Chemical Engineering and Analytical Science, University of Manchester, Manchester M13 9PL, UK 
interpretation of the results obtained from different methods helps to better understand the complex behavior of the porous material.

The issue has 12 papers in total, which illustrate notable recent developments in the field. In the first paper, Nezhad et al. (2019) describe a series of laboratory-scale model tests on flow and transport through packed beds of spheres, studying effects of grain size and flow rate on the flow regimes and dispersion process. The model tests show that more complex flow behavior is generated in the porous beds with finer particles and resulted in solute transport process with higher rate of dispersion.

Meng and Li (2019) present a surrogate model using sparse polynomial chaos expansion method to simulate unconfined groundwater flow in heterogeneous subsurface domain. They study efficiency of the polynomial chaos expansion method for quantifying uncertainty associated with nonlinear unconfined flow in porous media with spatially random hydraulic conductivity, and subjected to variable discharge of recharge flow rates. The methodology used in this paper may be later employed for simulation of flow in real confined and unconfined domains, potentially leading to future improvements and savings in practice. Gadd et al. (2019) examine the reliability of manifold learning method in the form of local tangent space alignment to develop surrogate models for groundwater flow. In the next paper, Crevillén-García et al. (2019) present a reduced-order stochastic model developed based on Gaussian process modelling approaches and simultaneous dimension reduction technique to reduce dimensionality of the hydraulic parameters of heterogeneous domain. They show how to significantly reduce the dimensionality of the high-dimensional input and output spaces of groundwater flow governing equations while retaining the qualitative features of the original model. Xue et al. (2019) present a framework using truncated power variogram model to quantify the uncertainty propagation in analysis of steady-state flow in saturated porous media. Considering the significance of studying multi-phase flow with the interest of complex reservoir simulations, Fuks et al. (2019) study uncertainty propagation in multi-phase flow in porous media and focus on estimating evolution of probability distribution of saturation in channelized porous systems.

Evolution of porous structure surface in space and time is also an interesting topic for design of reactive water treatment filters. Santisukkasaem and Das (2019) present a set of results on flow through permeable reactive barriers to study the effects of interactions between reactive materials surface and flow on permeability and effective performance of barriers. Gebäck and Heintz (2019) present a homogenization algorithm to compute macroscopic hydrological properties of periodic porous material and predict the effects of pore-scale osmosis phenomenon on the macroscale flow process.

Natural earth structures are mainly made of parallel or non-parallel layers, and this poses spatial variation in their hydro-mechanical properties and will affect the rate of fluid flow through the structures (Nezhad et al. 2013). Chiachío et al. (2019) present a new theoretical model based on Dupuit-Forchheimer theory for prediction of water table level in unconfined multilayered sloping systems with an unlimited number of non-parallel layers. Le et al. (2019) study the effects of spatial variability of soils on failure of slopes. They use stochastic analyses to predict probabilistic failure of slopes. They present correlation between spatial variability of hydrological properties and mechanical performance of earth structures, which is a popular topic among the researchers and practitioners interested in enhancing stability of flood embankments. The effects of layered structure of porous media on heat transfer and entropy generation of nanofluid are investigated by Asiaei et al. (2019). Their investigations and results provided new insights into the design of industrial nanoscale porous foams. Babaei (2019) presents a numerical study to evaluate the effects of uncertainties in reliable 
specification of reservoir boundary conditions on accurate performance prediction of multipurpose underground reservoirs.

In summary, this special issue contains papers that study the random behavior of porous media and flow mechanism through them using various experimental, numerical and analytical methods. It offers a wide-ranging overview of the present state of the science in the field. We expect that this special issue provides a useful resource for researchers and practitioners interested in the current advancements of the subject and hopefully it demystifies some of the complex science behind important open problems that are currently under investigation. We would like to thank all the authors, reviewers and our Editorial Advisory Panel members who have contributed greatly to the success of this themed issue. We hope that you enjoy reading the papers.

\section{References}

Asiaei, S., Zadehkafi, A., Siavashi, M.: Multi-layered porous foam effects on heat transfer and entropy generation of nanofluid mixed convection inside a two-sided lid-driven enclosure with internal heating. Transp. Porous Media (2019). https://doi.org/10.1007/s11242-018-1166-3

Babaei, M.: Integrated carbon sequestration-geothermal heat recovery: performance comparison between open and close systems. Trans. Porous Media (2019). https://doi.org/10.1007/s11242-018-1042-1

Chiachío, J., Chiachío, M., Sankararaman, S., Prescott, D.: A Bayesian assessment of an approximate model for unconfined water flow in sloping layered porous media. Transp. Porous Media (2019). https://doi. org/10.1007/s11242-018-1094-2

Crevillén-García, D., Leung, P.K., Rodchanarowan, A., Shah, A.A.: Uncertainty quantification for flow and transport in highly heterogeneous porous media based on simultaneous stochastic model dimensionality reduction. Transp. Porous Media (2019). https://doi.org/10.1007/s11242-018-1114-2

Dagan, G., Neuman, S.P.: Subsurface Flow and Transport: A Stochastic Approach. Cambridge University Press, Cambridge (1997)

Fuks, O., Ibrahima, F., Tomin, P., Tchelepi, H.A.: Analysis of travel time distributions for uncertainty propagation in channelized porous systems. Transp. Porous Media (2019). https://doi.org/10.1007/s11242$018-1052-\mathrm{z}$

Gadd, C., Xing, W., Nezhad, M.N., Shah, A.A.: A surrogate modelling approach based on nonlinear dimension reduction for uncertainty quantification in groundwater flow models. Transp. Porous Media (2019). https://doi.org/10.1007/s11242-018-1065-7

Gebäck, T., Heintz, A.: A pore scale model for osmotic flow: homogenization and lattice Boltzmann simulations. Transp. Porous Media (2019). https://doi.org/10.1007/s11242-017-0975-0

Le, T.M.H., Sanchez, M., Gallipoli, D., Wheeler, S.: Probabilistic study of rainfall-triggered instabilities in randomly heterogeneous unsaturated finite slopes. Transp. Porous Media (2019). https://doi.org/10.1007/ s11242-018-1140-0

Meng, J., Li, H.: Efficient uncertainty quantification for unconfined flow in heterogeneous media with the sparse polynomial chaos expansion. Transp. Porous Media (2019). https://doi.org/10.1007/s11242-0170974-1

Nezhad, M.M., Javadi, A.A., Al-Tabbaa, A., Abbasi, F.: Numerical study of soil heterogeneity effects on contaminant transport in unsaturated soil (model development and validation). Int. J. Numer. Anal. Methods Geomech. 37(3), 278-298 (2013)

Nezhad, M.M., Javadi, A.A.: Stochastic finite-element approach to quantify and reduce uncertainty in pollutant transport modeling. J. Hazard. Toxic Radioact. Waste 15(3), 208-215 (2011)

Nezhad, M. M.; Stochastic Finite Element Modeling of Flow and Solute Transport in Dual Domain System, Ph.D. thesis, University of Exeter, UK (2010)

Nezhad, M.M., Rezania, M., Baioni, E.: Transport in porous media with nonlinear flow condition. Transp. Porous Media (2019). https://doi.org/10.1007/s11242-018-1173-4

Riva, M., Guadagnini, A., Neuman, S.P., Franzetti, S.: Radial flow in a bounded randomly heterogeneous aquifer. Transp. Porous Media 45(1), 139-193 (2001)

Santisukkasaem, U., Das, D.B.: A non-dimensional analysis of permeability loss in zero-valent iron permeable reactive barrier (PRB). Transp. Porous Media (2019). https://doi.org/10.1007/s11242-018-1096-0 
Xue, L., Li, D., Nan, T., Wu, J.: Predictive assessment of groundwater flow uncertainty in multiscale porous media by using truncated power variogram model. Transp. Porous Media (2019). https://doi.org/10.1007/ s11242-018-1071-9

Publisher's Note Springer Nature remains neutral with regard to jurisdictional claims in published maps and institutional affiliations. 